\title{
Research on Model and Algorithm of Laying Interceptor Projectile in Restricted Area
}

\author{
Lei Ren ${ }^{1}$ and Yue Jia ${ }^{2}$ \\ ${ }^{1}$ Graduate Management Group Doctor team, Dalian Naval Academy, Dalian 116018, China \\ ${ }^{2}$ Department of Underwater Weaponry and Chemical Defense, Dalian Naval Academy, Dalian 116018, China
}

\begin{abstract}
Aiming at the problem of acoustic compatibility existing in the integrated use of soft and hard torpedo defense weapons, put forward the solution of laying suspended interceptor projectile in restricted area. According to the different types of torpedo guidance, respectively established mathematical model for suspended interceptor projectile laying in restricted area, and optimize the internal function and the constraint conditions, give the calculation process of optimal result using complex algorithms, verify the correctness of the model and algorithm through examples, provides a theoretical basis for solving the optimization problem of suspended interceptor projectile laying in restricted area.
\end{abstract}

Keywords-suspended interceptor projectile; restricted area; laying model; complex algorithm

\section{INTRODUCTION}

Torpedo threat is one of the most serious safe problems for surface ship. With the ability of target recognition and intelligent degree increasing, simply rely on deception and interference of soft killing methods have been difficult to ensure ship safety, must completely destroy the target, in order to improve the survivability of warship, which requires the integrated use of hard and soft torpedo defense weapons, the most typical is the suspended acoustic decoy and suspended interceptor projectile ${ }^{[1]}$.

However, from the current research situation, research for their comprehensive use is limited to how to use the "soft decoy" to improve the probability of "hard kill", without considering the adverse effects of between hard and soft weapon $^{[2]}$. In fact, the response signals produced by the decoy are likely to affect the normal use of the suspended interceptor projectile. Considering the general situation, the surface ship first launches the suspended acoustic decoy, then launches the suspended interceptor projectile. In order to solve the acoustic compatibility problem between them, it is necessary to limit the laying area of interceptor projectile according to the layout of acoustic decoy ${ }^{[3]}$.

So, in order to maximize combat capability of comprehensive using the suspended acoustic decoy and the suspended interceptor projectile, how to establish suspended interceptor projectile laying model in restricted area, and seek a fast and accurate algorithm, has become a new problem must be solved.

\section{LAyout Model IN Restricted AREA}

\section{A. Laying Process Summary}

As shown in Fig. I, take the ship's position when torpedo alarm as the origin, and take the ship's heading when torpedo warning as the $\mathrm{X}$ axis forward, establish the right angle coordinate system. the torpedo alarm bear is the $B$, alarm angle is the $Q_{m}$. Considering if the torpedo's distance is short, the torpedo may have found surface ships, soft confrontation weapon is difficult to play a role, so here only consider the torpedo distance is relatively far. The torpedo distance information is difficult to accurately measure, only can estimates its range, according to working performance of the torpedo alarm sonar and hydrological condition of current sea.

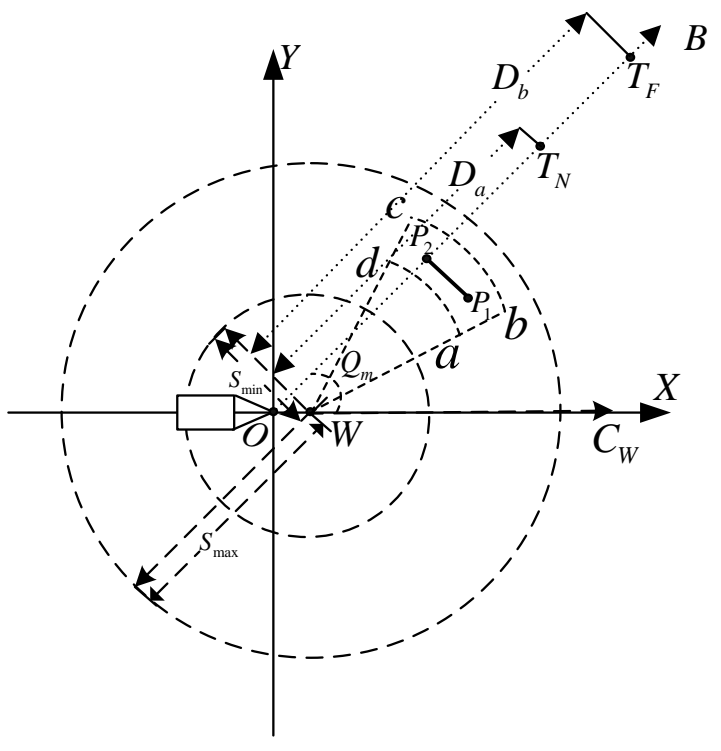

FIGURE I. LAYING PROCESS SKETCH MAP

Assuming that in the current hydrological condition, the nearest torpedo alarm distance is $D_{a}$, and the farthest torpedo alarm distance is $D_{b}$, the nearest and farthest torpedo possible positions are $T_{N}$ and $T_{F}$ respectively. When the incoming torpedo is found, the ship first launches the suspended acoustic decoy, and then it reaches the point $W$ after launching, and then launches the suspended interceptor projectile. It is assumed that the maximum range of the suspended interceptor 
projectile is $S_{\max }$, and the minimum range is $S_{\min }$. The suspended interceptor projectile is deployed by the "bilateral datum point", that is the two endpoints of the interception array are determined first, and then all the suspended interceptor projectile are placed on the line segments at a uniform interval according to the number of the interceptor projectile deployed $^{[4]}$. Once the incoming torpedo passes through this area, the suspended interceptor projectile will detonate and destroy the target torpedo. Due to the influence of the first launched suspended acoustic decoy, there is restricted area for the placement of the suspended interceptor projectile. Using firing direction and range to define the restricted area is the most convenient. So, here use the method described in the fifth references, assuming that the suspended interceptor projectile placed restricted area can be represented by a fan ring " abcd ", the center of the circle is the launching position $W$. In fact, the restricted area is given by the operational command system in actual combat, even if the area are not the fan ring, so can also take the largest fan ring which is contained by the actual region as the theoretical restricted area, then can solve the problem by the optimization model and algorithm in this paper.

\section{B. Laying Mathematics Model}

Generally, the intercepting targets of soft and hard torpedo defense weapons are acoustic homing or wire guided + acoustic homing torpedo. The characteristics of torpedo trajectory of these two guidance modes are different. The mathematical model of launching is established respectively. To obtain the maximum intercept probability is the optimization objective of the models.

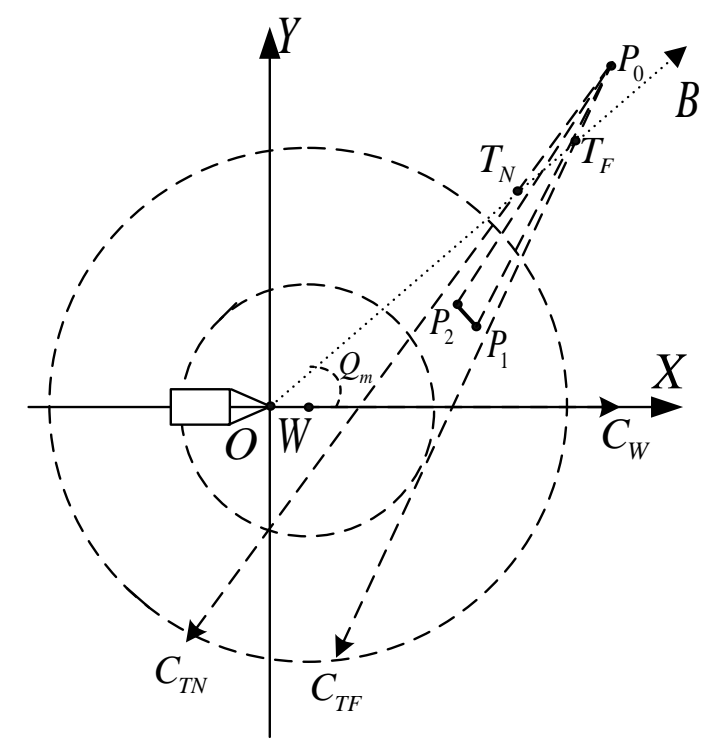

FIGURE II. INTERCEPT ACOUSTIC HOMING TORPEDO SKETCH MAP

As shown in Fig. II, if the incoming torpedo is an acoustic homing torpedo, its course is obtained by formula (1) and (2) ${ }^{[6]}$ :

$$
\left\{\begin{array}{l}
C_{T}=C_{W}+Q_{m}-180+\varphi_{a} \\
\varphi_{a}=\varphi-\beta
\end{array}\right.
$$

$$
\left\{\begin{array}{l}
m=\frac{V_{W}}{V_{T}} \\
\varphi=\arcsin \left(m \cdot \sin Q_{m}\right) \\
\beta=\arcsin \frac{R_{a} \cdot \sin \varphi}{D_{s}}
\end{array}\right.
$$

Where: " $C_{W}$ ” is the ship's course, " $\varphi_{a}$ " is torpedo firing advance angle, “ $V_{T}$ ” is torpedo's speed, “ $V_{W}$ ” is ship's speed, “ $D_{s}$ ” is target's distance at the time of weapon launch, " $R_{a}$ ” is torpedo's homing distance, and $m, \varphi, \beta$ are the intermediate variables.

By formula (1) and (2), it is known that when $R_{a}, C_{W}, Q_{m}$ are unchanged, $C_{T}$ is the function concerned $D_{s}$. Therefore, as shown in Fig. II , when torpedo alarm, the nearest torpedo's position is $T_{N}$, it's course is $C_{T N}$, the farthest torpedo's position is $T_{F}$, it's course is $C_{T F}$, and $C_{T N} \neq C_{T F}$, that is this two course must intersect, assuming that the intersection is $P_{0}$.

In order to obtain the maximum interception probability, we must make the intercept line $P_{1} P_{2}$ between the angle $\angle T_{N} P_{0} T_{F}$, and make the angle $\angle P_{1} P_{0} P_{2}$ as large as possible. So we can get the formula (3) and (4).

$$
\begin{aligned}
& f_{\text {max }}\left(P_{1}, P_{2}\right)=\angle P_{1} P_{0} P_{2}
\end{aligned}
$$

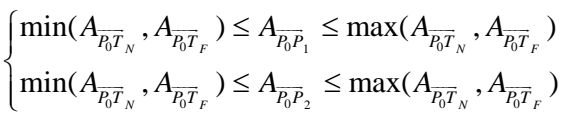

Where: $A_{P_{0} P_{1}}, A_{P_{0} P_{1}}, A_{P_{0} T_{N}}, A_{P_{0} T_{F}}$ are the true value orientation of $P_{1}, P_{2}, T_{N}, T_{F}$ relative $P_{0}$ respectively.

As shown in Fig.III, if the incoming torpedo is the wire guide + acoustic homing torpedo, the torpedo course is determined by formula (5) ${ }^{[7]}$ :

$$
\left\{\begin{array}{l}
C_{T}=C_{w}+Q_{m}-180^{\circ}+\varphi_{a} \\
\varphi_{a} \approx 0.8 \arcsin \left(\frac{V_{w}}{V_{T}} \sin Q_{m}\right)
\end{array}\right.
$$

According to the formula (5), the torpedo course is only related to the ship's course, the alarm angle and the ship's speed, but it has nothing to do with the alarm distance. Therefore, when torpedo warning sonar alarm, the torpedo course of the nearest position and the farthest position are the same 
theoretically, that is $C_{T}=C_{T N}=C_{T F}$, the actual torpedo course may be distributed between $C_{T N}$ and $C_{T F}$.

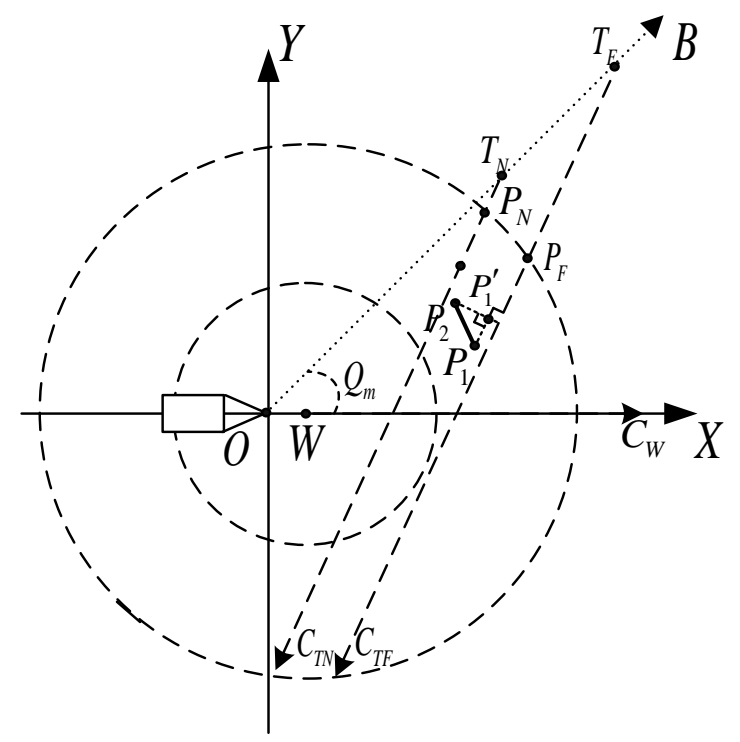

FIGURE III. INTERCEPT WIRE GUIED + ACOUSTIC HOMING TORPEDO SKETCH MAP

Similarly, in order to obtain the maximum interception probability, we must make the intercept line $P_{1} P_{2}$ between the near and far torpedo course line, and make the ends of $P_{1}, P_{2}$ in the $C_{T}$ normal projection line segment as long as possible. So we can get the formula (6) and (7).

$$
\begin{gathered}
f_{\max }\left(P_{1}, P_{2}\right)=\left|P_{1} P_{2}\right| \cdot \cos \theta \\
\left\{\begin{array}{l}
D_{\overline{P_{1} l_{N}}} \leq D_{\overline{I_{N} l_{F}}} \\
D_{\overline{P_{1} l_{F}}} \leq D_{\bar{l}_{N} l_{F}} \\
D_{\overline{P_{2} l_{N}}} \leq D_{\overline{l_{N} l_{F}}} \\
D_{\overline{P_{2} l_{F}}} \leq D_{\bar{l}_{N} l_{F}}
\end{array}\right.
\end{gathered}
$$

Where: $P_{1} P_{2}$ is the distance between $P_{1}$ and $P_{2} ; \theta$ is the sharp angle in the direction of $P_{1} P_{2}$ and $C_{T}$.

$$
D_{\overline{P_{1} I_{N}}}, D_{\overline{P_{1} l_{F}}}, D_{\overline{P_{2} l_{N}}}, D_{\overline{P_{2} l_{F}}} \text { are the distance from } P_{1}, P_{2} \text { to }
$$
the nearest, farthest torpedo course line respectively, $D_{\bar{I}_{N_{F} I_{F}}}$ is the distance from the nearest torpedo course line to the farthest torpedo course line.

$$
\left\{\begin{array}{l}
S_{\text {min }} \leq\left|W P_{1}\right| \leq S_{\text {max }} \\
S_{\text {min }} \leq\left|W P_{2}\right| \leq S_{\text {max }} \\
D_{\text {min }} \leq\left|W P_{1}\right| \leq D_{\max } \\
D_{\text {min }} \leq\left|W P_{2}\right| \leq D_{\max } \\
B_{\text {min }} \leq A_{\overline{W P_{1}}} \leq B_{\text {max }} \\
B_{\text {min }} \leq A_{\overline{W P_{2}}} \leq B_{\text {max }}
\end{array}\right.
$$

In consideration of the range limitation of the suspended interceptor projectiles and restricted area conditions, the above the mathematical models described by formula (3), (4), and formula (6), (7) must be added (8) as a constant constraint condition.

Where: $\left[S_{\min }, S_{\max }\right]$ is the firing range of the suspended interceptor projectile, and the $\left[D_{\min }, D_{\max }\right]$ and $\left[B_{\min }, B_{\max }\right]$ are the given distance range and azimuth range of the restricted area. $\left|W P_{1}\right|,\left|W P_{2}\right|$ are the distances from $W$ to $P_{1}, P_{2}$, respectively. $A_{\overrightarrow{W P_{1}}}$ and $A_{\overrightarrow{W P_{2}}}$ were true value orientation of $P_{1}$, $P_{2}$ relative to $W$ respectively.

\section{Complex Algorithm}

1) The basis and reason of algorithm selection.

If the mathematical model is directly solved according to the formula (3), (4) and (6) (8), the calculation process will be very complex. In order to solve this optimization problem, complex optimization method can be used according to the characteristics of its objective function and constraint conditions.

The complex method is an extension of the simplex method, and it is a direct algorithm for inequality constraints. Its basic idea is feasible in the design space within the structure with $\mathrm{k}$ vertices of the polyhedron, according to vertex function value line, find the best point and minimum value function, and the worst point and maximum value function, and calculate the reflection point of the worst point, common reflection point is better than the worst point, so the reflection point instead of the dead, a new complex, so adjust the vertices of the polyhedron, the polyhedron is closer to the advantage, finally search to the best points ${ }^{[8]}$.

The optimal model of P1 and P2 established above is actually an optimization model with 4 design variables and constraints on the plane two point coordinate, which satisfies the condition of using the complex method in form. However, if the algorithm is applied directly, the variable dimension and the expression of each function are still complex, and the convergence degree of the algorithm must be slower. In order to improve the calculation speed, the objective function and the constraint condition of the model need to be optimized.

\section{2) Optimization of objective function}

a) The incoming torpedo is an acoustic homing torpedo

We can see from Fig. II, when interception line between the angle $\angle T_{N} P_{0} T_{F}$ which is constituted by nearest and farthest torpedo course, the angle $\angle P_{1} P_{0} P_{2}$ is equal to the angle 
$\angle T_{N} P_{0} T_{F}$ subtract the angle which is constituted by nearest and farthest torpedo course and $P_{0} P_{1}, P_{0} P_{2}$ respectively, i.e.:

$$
f_{\text {max }}\left(P_{1}, P_{2}\right)=\angle T_{N} P_{0} T_{F}-\angle T_{N} P_{0} P_{1}-\angle T_{F} P_{0} P_{2}
$$

Where: the angle $\angle T_{N} P_{0} T_{F}$ can be obtained by calculation with nearest and farthest torpedo course. $\angle T_{N} P_{0} P_{1}, \angle T_{N} P_{0} P_{1}$ are independent of each other. Therefore, the optimization problem is transformed into constraints derived from $P_{1}$ and $P_{2}$, make the angle between the $P_{0} P_{1}$ and the nearest torpedo course as small as possible, and make the angle between the $P_{0} P_{2}$ and the farthest torpedo course as small as possible.

Suppose that the azimuth of $P_{1}$ is $B_{P_{1}}$, the distance of $P_{1}$ is $D_{P_{1}}$, the azimuth of $P_{2}$ is $B_{P_{2}}$, the distance of $P_{2}$ is $D_{P_{2}}$, then the objective function can be described by formula (10) and (11):

$$
\begin{gathered}
f \min \left(B_{P_{1}}, D_{P_{1}}\right)=\frac{D_{P_{1}} \cdot \sin B_{P_{1}}-y_{p_{0}}}{D_{P_{1}} \cdot \cos B_{P_{1}}-x_{p_{0}}} \\
f \min \left(B_{P_{2}}, D_{P_{2}}\right)=\frac{D_{P_{2}} \cdot \cos B_{P_{2}}-x_{p_{0}}}{D_{P_{2}} \cdot \sin B_{P_{2}}-y_{p_{0}}}
\end{gathered}
$$

b) The incoming torpedo is an wire guied + acoustic homing torpedo

We can see from Fig. III, when interception line $P_{1} P_{2}$ between the nearest torpedo course and the farthest torpedo course, The length of the projection line of $P_{1} P_{2}$ in the normal direction of $C_{T}$ is equal to the distance $D_{\overline{l_{N} I_{F}}}$ between the nearest and farthest torpedo course subtracts the distance $D_{\overline{P_{1} I_{N}}} D_{\overline{P_{2} l_{F}}}$, which are from $P_{1}, P_{2}$ to the nearest and farthest torpedo course respectively, i.e.:

$$
f_{\max }\left(P_{1}, P_{2}\right)=D_{\overline{l_{N} l_{F}}}-D_{\overline{P_{1} l_{N}}}-D_{\overline{P_{2} l_{F}}}
$$

Where: the angle $D_{\overline{I_{N} l_{F}}}$ can be obtained by calculation with nearest and farthest torpedo course. $D_{\overline{P_{1} I_{N}}}, D_{\overline{P_{2} l_{F}}}$ are independent of each other. Therefore, the optimization problem is transformed into constraints derived from $P_{1}$ and $P_{2}$, make the distance between $P_{1}$ and the nearest torpedo course as small as possible, make the angle between $P_{2}$ and the farthest torpedo course as small as possible.

According to the formula of the distance from point to line, the objective function can be expressed by formula (13) and (14): $f \min \left(B_{P_{1}}, D_{P_{1}}\right)=\frac{\cot C_{T} \cdot D_{P_{1}} \cdot \cos B_{P_{1}}-D_{P_{1}} \cdot \sin B_{P_{1}}+\left(y_{T_{N}}-\cot C_{T} \cdot x_{T_{N}}\right)}{\sqrt{\cot C_{T}^{2}+1}}$

$f \min \left(B_{P_{2}}, D_{P_{2}}\right)=\frac{\cot C_{T} \cdot D_{P_{2}} \cdot \cos B_{P_{2}}-D_{P_{2}} \cdot \sin B_{P_{2}}+\left(y_{T_{N}}-\cot C_{T} \cdot x_{T_{N}}\right)}{\sqrt{\cot C_{T}^{2}+1}}$

After removing the constants, the formulas are simplified as (15) and (16):

$$
\begin{aligned}
& f \min \left(B_{P_{1}}, D_{P_{1}}\right)=\cot C_{T} \cdot D_{P_{1}} \cdot \cos B_{P_{1}}-D_{P_{1}} \cdot \sin B_{P_{1}} \\
& f \min \left(B_{P_{2}}, D_{P_{2}}\right)=\cot C_{T} \cdot D_{P_{2}} \cdot \cos B_{P_{2}}-D_{P_{2}} \cdot \sin B_{P_{2}}
\end{aligned}
$$

After a series of transformations, the original fourdimensional objective function can be reduced to two twodimensional objective functions to achieve simultaneous computation, which greatly improves the computational speed.

\section{3) Optimization of the constraints}

Compare the range of the weapon and the restricted area, The constant constraint condition is optimized and the 4 design variables are substituted. The formula (17), (18) can be obtained.

$$
\left\{\begin{array}{l}
\max \left(S_{\min }, D_{\min }\right) \leq D_{P 1} \leq \min \left(S_{\max }, D_{\max }\right) \\
B_{\text {min }} \leq B_{P 1} \leq B_{\text {max }}
\end{array}\right.
$$

$$
\left\{\begin{array}{l}
\max \left(S_{\min }, D_{\min }\right) \leq D_{P_{2}} \leq \min \left(S_{\max }, D_{\text {max }}\right) \\
B_{\min } \leq B_{P_{2}} \leq B_{\max }
\end{array}\right.
$$

For the functional constraints, the azimuth constraint in the Cartesian coordinate system (4) described by the slope constraint, which is shown in the formula (18) for the convenience of calculation.

$$
\left\{\begin{array}{l}
\min \left(k_{P_{0} T_{N}}, k_{P_{0} T_{F}}\right) \leq k_{P_{0} P_{1}} \leq \max \left(k_{P_{0} T_{N}}, k_{P_{0} T_{F}}\right) \\
\min \left(k_{P_{0} T_{N}}, k_{P_{0} T_{F}}\right) \leq k_{P_{0} P_{2}} \leq \max \left(k_{P_{0} T_{N}}, k_{P_{0} T_{F}}\right)
\end{array}\right.
$$

The 4 design variables are substituted into (19) and (7), and the formulas (20) and (21) are used as functional constraints.

$$
\left\{\begin{array}{l}
\min \left(k_{P_{0} T_{N}}, k_{P_{0} T_{F}}\right) \leq \frac{D_{P_{1}} \cdot \sin B_{P_{1}}-y_{p_{0}}}{D_{P_{1}} \cdot \cos B_{P_{1}}-x_{P_{0}}} \\
\leq \max \left(k_{P_{0} T_{N}}, k_{P_{0} T_{F}}\right) \\
\min \left(k_{P_{0} T_{N}}, k_{P_{0} T_{F}}\right) \leq \frac{D_{P_{2}} \cdot \sin B_{P_{2}}-y_{P_{0}}}{D_{P_{2}} \cdot \cos B_{P_{2}}-x_{P_{0}}} \\
\leq \max \left(k_{P_{0} T_{N}}, k_{P_{0} T_{F}}\right)
\end{array}\right.
$$




$$
\left\{\begin{array}{l}
\cot C_{T} \cdot D_{P_{1}} \cdot \cos B_{P_{1}}-D_{P_{1}} \cdot \sin B_{P_{1}} \leq \\
D_{I_{N_{F} l_{F}}} \cdot \sqrt{\cot C_{T}^{2}+1}-\left(y_{T_{N}}-\cot C_{T} \cdot x_{T_{N}}\right) \\
\cot C_{T} \cdot D_{P_{1}} \cdot \cos B_{P_{1}}-D_{P_{1}} \cdot \sin B_{P_{1}} \leq \\
D_{I_{N} I_{F}} \cdot \sqrt{\cot C_{T}^{2}+1}-\left(y_{T_{F}}-\cot C_{T} \cdot x_{T_{F}}\right) \\
\cot C_{T} \cdot D_{P_{2}} \cdot \cos B_{P_{2}}-D_{P_{2}} \cdot \sin B_{P_{2}} \leq \\
D_{I_{N_{N} I_{F}}} \cdot \sqrt{\cot C_{T}^{2}+1}-\left(y_{T_{N}}-\cot C_{T} \cdot x_{T_{N}}\right) \\
\cot C_{T} \cdot D_{P_{2}} \cdot \cos B_{P_{2}}-D_{P_{2}} \cdot \sin B_{P_{2}} \leq \\
D_{\overline{I_{N} l_{F}}} \cdot \sqrt{\cot C_{T}^{2}+1}-\left(y_{T_{F}}-\cot C_{T} \cdot x_{T_{F}}\right)
\end{array}\right.
$$

\section{CALCULATION METHOD}

\section{A. Determination of Initial Feasible Value}

In the calculation of initial need to enter a group to meet the constraints of the value process, because the initial conditions are difficult to directly meet the function value is given, so the use of randomly generated data from constant constraints in the constraint conditions into the judgment way to obtain the feasible initial data.

\section{B. Calculation Process}

The calculation of laying model is mainly to find the optimal layout datum point, and the calculation flow is shown in the Fig. IV.

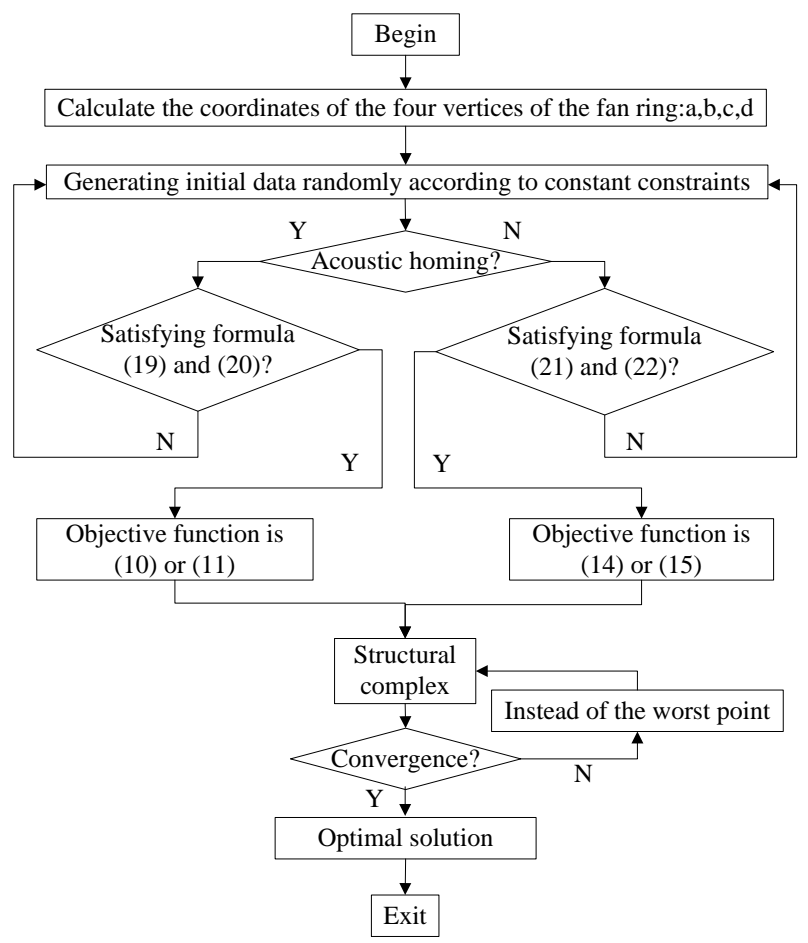

FIGURE IV. LAYING DATUM POINT CALCULATE PROCES

The bilateral datum point of Intercept array can be determined, according to the length of $P_{1} P_{2}$ and weapon distribution quantity, calculate the interval of suspended interceptor projectile, then calculate the position of suspended interceptor projectile according the direction of the intercept array.

\section{Determination of Algorithm Parameters}

In the calculation process of complex optimization algorithm, several parameters must be determined: the reflection coefficient: alpha , the number of allowed iterations: $k$, and the requirement of control accuracy: eps. Here the general value, i.e.: $a l p h a=1.3, k=1000$, and the control precision is related to the accuracy of the algorithm, and the computational time required to take into consideration, so eps $=0.01^{[9]}$.

\section{EXAMPLE VERIFICATION}

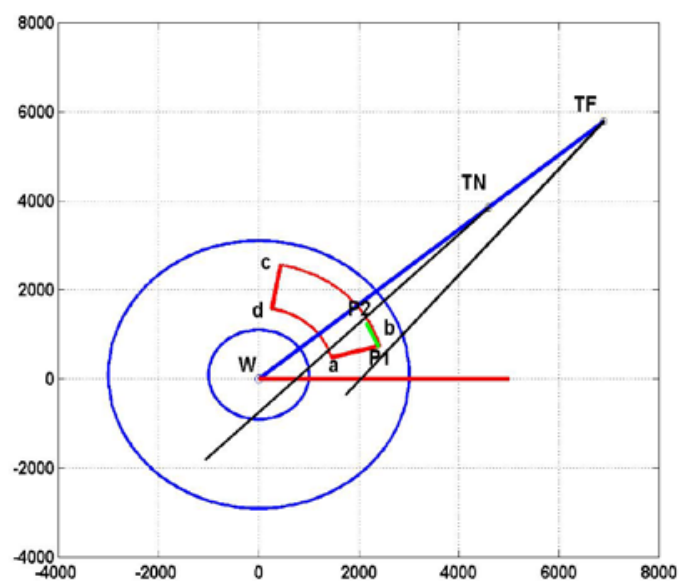

FIGURE V. INTERCEPT ACOUSTIC HOMING TOEPESO CALCULATION RESULT

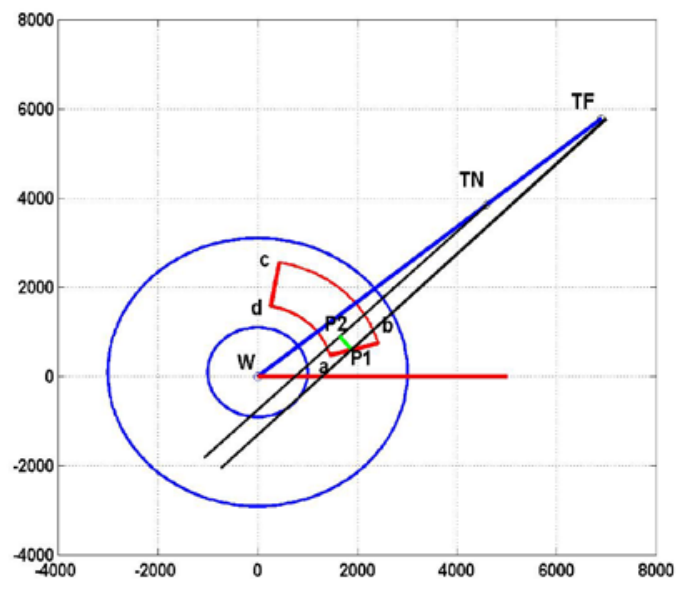

FIGURE VI. INTERCEPT WIRE GUIDE + ACOUSTIC HOMING TOEPESO CALCULATION RESULT

According to the above calculation process using MATLAB programming calculation and drawing, calculation for acoustic homing and wire + two acoustic homing torpedo weapon cloth put the position of the results shown in Fig. V, shown in Fig.VI. The initial conditions of calculation are as follows: $C_{W}=90^{\circ}, Q_{m}=-40^{\circ}$, warning hydrological 
condition is medium. The azimuth range of the suspended interceptor projectile is $\left[10^{\circ}, 75^{\circ}\right]$. The distance range of the suspended interceptor projectile is $[1500 \mathrm{~m}, 2500 \mathrm{~m}]$.

From Fig. V and Fig. VI, we can see that the optimal solution, which is calculated accordance with the above optimization model and complex algorithms, can satisfy the requires of suspended interceptor projectile laying in the restricted area. That is maximize the probability of interception.

\section{CONCLUSION}

In the regional conditions, the difficulties in calculation of suspended interceptor projectile distribution is considered likely to torpedo moving area and suspended interceptor projectile cloth limited area, in this case by using the above optimization deployment model and complex algorithm, combined with the compared by analytical calculation and logic judgment the algorithm can avoid a lot of logic, judgment, which is difficult to achieve with other nonlinear algorithm. However, as seen from Fig. V, Fig. VI can also, when meet the optimal interception probability of the highest goal of optimal solution is not unique, the algorithm can automatically be judged from the target of torpedo from the nearest optimal solution, which reached as far as soon as possible to intercept the attacking torpedo, which is the next step for the improvement and research needs the model and algorithm of direction.

\section{REFERENCE}

[1] Chen Chunyu, Anti Torpedo Technology, 1rd ed. Beijing: National Defense IndustryPress, 2006, pp.38-59.

[2] Ren Lei, Jia Yue, Li Wenzhe, "Research on Comprehensive Scheme of Shipboard Hard and Soft Torpedo Defense Weapons Against Intelligent Torpedoes” Acta Armamentarii. China, vol. 6, pp. 2336-2341, December 2015.

[3] Jia Yue, Yao Fengliang, Ding Bei, "Modeling and Simulation of Cooperative Defense Against Acoustic Homing Torpedo with Hovering Acoustic Decoy and Hovering Depth Charge Array" Torpedo Technology. China, vol. 3, pp. 231-233,March 2011.

[4] Xu Liang, Xie Guoxin, Zhao Zhijun, "Research on Suspended Depthcharge Torpedo Intercept Method" Mine Warfare and Ship Protection. China, vol. 4, pp. 50-53, April 2010.

[5] Zhao Xiangtao, Li Wenzhe, Kou Zhu, "Analysis on Shooting Area for Intercepting Torpedo of Suspended Depth Charg” Sichuan Ordnance Journal. China, vol. 11, pp. 20-22, November 2011.

[6] Lin Xianjie, Jia Yue, Zhao Xiangtao, "Ship Evasive Strategy while Releasing Suspended Depth Charge Againgst Acoustic Homing Torpedo” torpedo technology. China, vol. 16, pp. 51-53, April 2008 .

[7] Yao Fengliang, Jia Yue, Ding Bei, "Research on the Strategy of Intercepting Wire-guide Torpedo Using Hovering Depth Charge" Command Control and Simulation. China, vol. 5, pp. 24-27, May 2010 .

[8] Xu Yadong, Qian Fang Lin, "Optimization of Composite Material Barrel Based on Complex Method” Journal of Nanjing University of Science and Technology. China, vol. 6, pp. 635-638, June 2005 .

[9] Chen Hui, Zhan Shaohua, "Optimization Design of Two Cylindrical Gear Reducer Based on Complex Method of MATLAB” coal mineg machinery. China, vol. 8, pp. 31-33, August 2011. 